\title{
Towards mimicking cold exposure
}

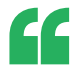

12,13-diHOME activates brown adipose tissue fuel uptake
A lipid, 12,13-dihydroxy-9Zoctadecenoic acid (12,13-diHOME), that is released in response to cold activates brown adipose tissue, according to a new paper published in Nature Medicine.

Research in the past few years has focused on activating brown adipose tissue as a potential treatment for obesity and diabetes mellitus. So far, cold exposure has proved to be the most effective method of activating brown adipose tissue; however, cold exposure is not a practical therapy as it is time consuming and uncomfortable for most people. This problem led a group of researchers to search for cold mimetics that could be used to activate brown adipose tissue.

The investigators exposed human volunteers to $1 \mathrm{~h}$ of cold at $14^{\circ} \mathrm{C}$ and then collected blood samples. Liquid chromatography-tandem

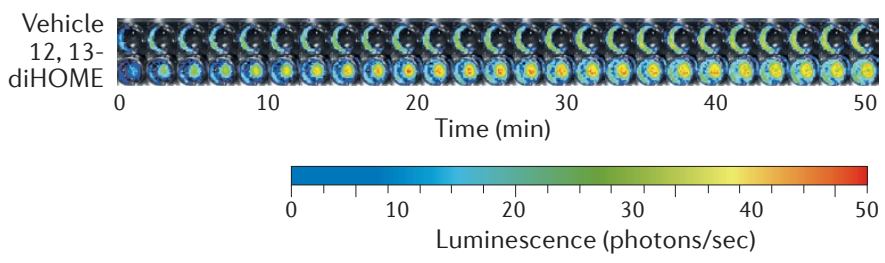

In this representative image, in vitro differentiated brown adipocytes that constitutively express firefly luciferase were treated with 100 uM 12,13-diHOME (bottom row) or vehicle (top row) for $15 \mathrm{~min}$. Fatty acid uptake was measured by adding fatty acid-luciferin, a D-luciferin-conjugated fatty acid that is reduced intracellularly, freeing the D-luciferin from the fatty acid and allowing luciferase-mediated oxidation to be measured by bioluminescence. Each well was imaged repeatedly for approximately $50 \mathrm{~min}$. Image courtesy of M.D. Lynes and Y.-H. Tseng. mass spectrometry was used to measure the concentrations of 88 lipids in these samples. The levels of three lipids were increased after the cold exposure and that of one, 12,13-diHOME, was increased in all participants. Measuring radiolabelled glucose uptake revealed that the concentration of 12,13-diHOME correlated with the activity of brown adipose tissue. The plasma concentration of 12,13-diHOME was also negatively correlated with BMI, insulin resistance and fasting plasma levels of insulin and glucose; however, no correlation was found between concentrations of 12,13-diHOME and levels of $\mathrm{HbA}_{1 \mathrm{c}}$ and C-reactive protein.

Next, the researchers injected mice with $1 \mu \mathrm{g} / \mathrm{kg}$ of 12,13 -diHOME and exposed them to cold. Compared with mice given vehicle, those who received 12,13-diHOME were protected from the drop in body temperature during the cold challenge. Importantly for its potential use as a thera peutic, administration of 12,13-diHOME had no long-term effects on blood pressure or pulse. To test its therapeutic potential, the investigators gave mice with diet-induced obesity a daily injection of $10 \mu \mathrm{g} / \mathrm{kg}$ of 12,13-diHOME for 2 weeks. The treatment decreased circulating levels of triglycerides but had no effect on body weight or glucose tolerance.
In addition, the researchers found that 12,13-diHOME supplementation increased the uptake of lipids, fatty acids and glucose into brown adipose tissue in mice. "12,13-diHOME supplementation activated substrate uptake into brown adipose tissue where it could be oxidized and broken down," explain authors Yu-Hua Tseng and Matthew Lynes.

"Our results indicate that, in addition to serving as a biomarker for brown adipose tissue activation, 12,13-diHOME activates brown adipose tissue fuel uptake, thereby decreasing circulating triglyceride levels," comment Tseng and Lynes. The researchers note that more work needs to be done to elucidate the cellular mechanisms of action of 12,13-diHOME. "However, given that 12,13 -diHOME is a lipid mediator naturally produced in the body, we would like to test the therapeutic effects of 12,13-diHOME supplementation on individuals with dyslipidaemia or related metabolic disorders," conclude Tseng and Lynes.

Claire Greenhill

ORIGINAL ARTICLE Lynes, M. D. et al. The coldinduced lipokine 12,13-diHOME promotes fatty acid transport into brown adipose tissue. Nat. Med. http://dx.doi.org/10.1038/nm.4297 (2017) 